\title{
Welche Elemente charakterisieren die Osteopathie?
}

\author{
Jo Buekens
}

Jeder von uns hat unterschiedliche äußere Anregungen und innere Beweggründe, die dazu geführt haben, die Osteopathieausbildung in der letztendlich ausgewählten Art und an dem gewählten Ort begonnen zu haben. Zusätzlich gibt es die äußerst komplexe Geschichte der Osteopathie, aus der jeder Einzelne die unterschiedlichsten Definitionen und Schwerpunkte der Osteopathie herauslesen und für sich selbst betonen kann. Zwischen diesen vielen unterschiedlichen persönlichen und gruppengebundenen Interessen geht häufig der ursprüngliche Ansatz des Gründervaters komplett verloren. Inspiriert durch Michael A. Lanes’ Publikation aus dem Jahr 1918 möchte ich hier Stills grundlegenden philosophischen und wissenschaftlichen Ansatz als Ausgangspunkt zur Weiterentwicklung der Definition der Osteopathie nutzen.

\section{Was macht die Osteopathie einzigartig?}

Wenn wir A. T. Stills Denkschritte untersuchen, zeigt sich, dass nicht alle seine philosophischen Ideen und Prinzipien direkt der „Osteopathie“ als solcher zugeschrieben werden können. Daher sollte man über die wesentlichen Aspekte nachdenken, welche die Osteopathie von allen anderen medizinischen und therapeutischen Ansätzen unterscheidet. Obwohl der „alte Doktor“ die Meinung vertrat, dass bei Osteopathen der Tastsinn in einem sehr hohen Maß entwickelt sein und eine wesentliche Qualifikation eines erfolgreichen osteopathischen Arztes sein muss [1], sind unsere heilenden Hände nicht die entscheidenden Unterscheidungsmerkmale. Tatsächlich sind die magischen Hände auch für Chiropraktiker, Manualtherapeuten, Physiotherapeuten, Masseure und viele andere unverzichtbar.

Wie wäre es dann mit „panta rhei“, dem sog. freien Flüssigkeitsaustausch? Richtig: In der Osteopathie nutzen wir dieses Prinzip auch, um einen guten Teil unserer therapeutischen Ansätze zu erklären. Aber ist es das, was uns einzigartig macht? Ich fürchte nicht, weil dieses Prinzip auch in der Homöopathie, Akupunktur, im Reiki und bei anderen naturheilkundlichen Therapieverfahren und alternativmedizinischen Konzepten großgeschrieben wird.

Zusätzlich müssen wir uns eingestehen, dass viele der sog. philosophischen osteopathischen Säulen, auf die immer wieder in Interviews, Flyern und Websites verwiesen wird, eher Teile der medizinischen Grundphilosophie anzusehen sind, wie sie von Hippokrates, Sokrates und anderen griechischen Philosophen formuliert wurden.

Was also ist es, das die Osteopathie einzigartig macht? Was sind die Leitgedanken bei unserer Arbeit mit Patienten oder beim Studieren? Einen wichtigen Teil der Antwort gibt Still im Interview vom 28.11.1904, in dem er sagt: „Ich entschied um 1845, dass diese unbekannte Intelligenz, nennen Sie sie, wie Sie wollen, die allen Strukturen und Funktionen zugrunde liegt, vertrauenswürdig ist. Ihre Arbeit ist perfekt.“ [2]

\section{Apotheke Gottes}

Seine Argumentation ist in der heutigen Zeit noch aktuell: Denn kein Molekül oder Medikament, das entwickelt wurde, ist wirklich neu. Die meisten synthetisch hergestellten Medikamente sind „einfache“ Kopien von körpereigenen Molekülen. Dementsprechend entwickelte der alte Doktor einen praktischen Ansatz, mit dem er versuchte, die inneren Regulationskräfte zu stimulieren, sodass der Körper selbst seine Regulationssysteme starten oder verbessern konnte. Still nannte den Körper „Apotheke Gottes“.

Der wesentliche Unterschied zwischen der Medizin und der Osteopathie liegt darin, wie sie mit dem Angebot der Apotheke Gottes umgehen. Still formulierte: „Ich glaube, der Mensch machte den Fehler, als er damit begann, giftige Substanzen als Heilmittel für Krankheiten in das menschliche System zu injizieren, anstatt die Ge- 
setze der Schöpfung zu diesem Zweck anzuwenden. Hier ist der Punkt, an dem Osteopathie und Medizin getrennte Wege gehen.“ [3]

Es ist bekannt, dass Still in seiner Kommunikation viele Metaphern benutzte. Die Frage, die sich daraus ergibt, ist: Wie haben wir diese zu verstehen? Michael A. Lane, der 1928 den Distinguished Service Award der American Osteopathic Association erhielt [4], hatte eine überraschende, aber wissenschaftliche Deutung der Apotheken-Metapher. Für ihn war Still der Erste, der die innere Kraft zur Erhaltung und Wiederherstellung der Gesundheit mit dem allgemeinen Gesetz der Immunität verband, das Lane als das erste, für die Osteopathie bestimmende, Axiom definierte [5].

Still hatte von Anfang an die Beobachtung gemacht, dass der menschliche Körper durch den Kontakt mit Krankheitserregern nicht grundsätzlich krank wurde. Darüber hinaus bemerkte er, dass in einigen Fällen Patienten nach dem Auftreten einer Krankheit für eine Weile oder sogar ein ganzes Leben lang resistent zu sein schienen. So argumentierte er, dass er irgendwo irgendetwas in unserem Körper, im Blut, vor zerstörerischen Krankheitserregern bewahren müsse. Dass es einen natürlichen vererbten Widerstandsmechanismus geben müsse, eine Abwehr, die den Körper gegen die Krankheit reagieren lasse und es ihm ermögliche, sich zu erholen und sogar zu heilen. Erst 1889, 15 Jahre nachdem Still die Immunität und die Apotheke Gottes als wichtigste Axiome seiner Vorgehensweise definiert hatte, publizierte Paul Ehrlich die Theorie der Immunologie.

\section{Läsionen der Wirbelsäule}

Der Schlüssel zur Apotheke liegt im 2. Axiom: dem Gesetz der Wirbelsäulenläsion und dem entscheidenden ätiologischen Faktor. Still unterschied zwischen primären und sekundären Läsionen, die er wie folgt definierte [5]:

- Primäre Läsionen entstehen in der Wirbelsäule selbst und wirken reflexartig auf andere Körperteile.

- Sekundäre Läsionen entstehen in anderen Körperteilen und werden in der Wirbelsäule kompensiert.

Dieses Gesetz ist direkt mit einer der wichtigsten Körperstrukturen verbunden: dem Rückgrat. Um dies zu bekräftigen, schrieb Lane: „Für Still bedeutete Anatomie studieren, wahrzunehmen und zu sehen, dass das Rückgrat der Schlussstein des Körpers ist, und dass dieser königliche Palast des hoheitlichen Gewebes - dem Nerv - die Quelle andauernder Störungen des zirkulierenden Blutes war.“ [5]

Mit einem manuellen Ansatz untersuchte und behandelte der alte Doktor den erkrankten Körper, normalisierte die Läsionen und versuchte, die Körperabwehr und damit den Heilungsmechanismus zu stimulieren, sodass der
Normalzustand wiederhergestellt werden konnte. „Finde es, repariere es - und lass' es in Ruhe“, hieß es in diesem Zusammenhang, weil Still bewies, dass der Körper nach der Korrektur der Läsion in der Lage war, seinen Weg zur Immunität und Normalität zu finden.

\section{Freier Fluss der Körperflüssigkeit}

Das 3. Axiom führt uns schließlich zum Hauptziel der osteopathischen Behandlung - „The rule of the artery is supreme“ [5]. Es sagt uns: „Beseitige den Grund, der den Blutfluss stoppt oder hemmt oder den Nerv blockiert, welcher den Blutfluss kontrolliert, und das Blut selbst wird die Heilung bewirken." [5]

Zu Lebzeiten von Still galt es vielfach als völlig absurd, alle Krankheiten unter einem Leitprinzip zu betrachten. Und doch war es genau dieses Prinzip, das die Osteopathie revolutionär machte und immer noch macht. Wenn wir durch unseren Ansatz in der Lage sind, den freien Fluss der Körperflüssigkeiten und insbesondere des arteriellen Bluts wiederherzustellen, wird der Körper seine Gesundheit aufrechterhalten oder wiederherstellen.

Um dorthin zu gelangen, können viele therapeutische Verfahren genutzt werden. Sie reichen von der dynamischen Gewebemobilisierung über die Muskel-EnergieTechnik bis zur High-Velocity-Low-Amplitude oder der Bereitstellung eines Fulkrums, um die Funktion und Physiologie des Körpers so zu optimieren, dass die Apotheke Gottes funktionsfähig bleibt. Dabei sollten wir uns vor Augen halten, dass nicht unser Input wichtig ist, sondern die Art, wie der behandelte Körper mit diesem Input umgeht. Wenn die angewandte Behandlung die 3 grundlegenden Axiome gut erfüllt, gibt es keine Notwendigkeit für von außen zugeführte Moleküle, Nadeln, Potenzen oder magische Techniken.

Das ist die einzigartige und fabelhafte Idee, die A.T. Still hatte und welche die Osteopathie von allen anderen medizinischen Verfahren unterscheidet. In der Osteopathie werden gesundheitliche Probleme vom Körperinneren her gelöst, nicht von außen.

\section{Welches Körpergewebe ist typisch osteopathisch?}

An dieser Stelle ergibt sich aber noch eine sehr wichtige Frage: Welches Körpergewebe ermöglicht uns, diesen einzigartigen osteopathischen Weg zu gehen? Einmal mehr kommt die Antwort vom Begründer der Osteopathie, wenn er in seiner Autobiografie von 1908 die Osteopathie wie folgt definiert: „Osteopathie ist zusammengesetzt aus den 2 Wörtern osteo, was Knochen, und pathos, was Leiden bedeutet. Meine Überlegung war, dass der Knochen, ,Osteon', der Ausgangspunkt war, 


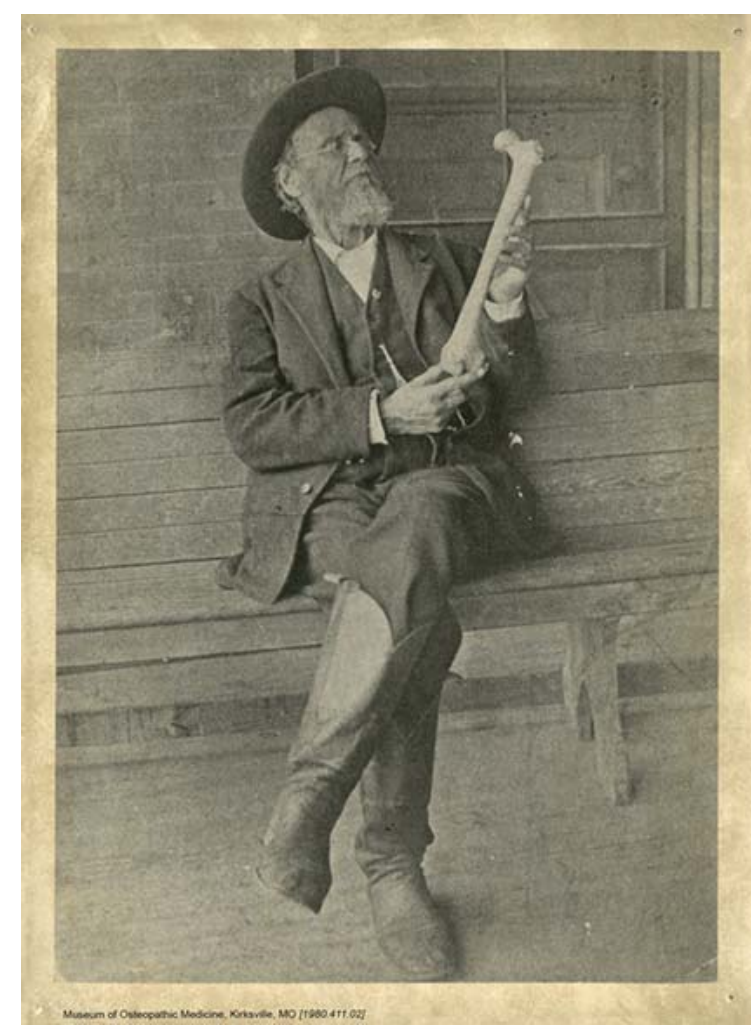

- Abb. 1 Andrew Taylor Still (1828-1917) begründete vor über 130 Jahren die Osteopathie. (@ Museum of Osteopathic Medicine, Kirksville [1980.411.02])

von dem aus ich die Ursache für pathologische Zustände feststellen konnte, und so kombinierte ich ,Osteo “ mit der ,Pathie‘ und hatte ein Ergebnis, die Osteopathie“ [6].

In dieser Definition redet Still nicht von Gelenken, sondern von Knochen. Viele bedeutende, hauptsächlich europäische Wissenschaftler waren damals von der Form und Struktur dieses einen mysteriösen Knochens fasziniert: vom Femur. Sie alle versuchten zu verstehen, warum diese kranähnliche Struktur von Femurkopf und Femurhals unter Last nicht brach. Durch die Form des Femurs wurde die gesamte Forschung ausgelöst, die zur Entdeckung der trabekulären Struktur und Architektur führte.

Da Still den Knochen als „Medium“ zur Bestimmung pathologischer Bedingungen definiert hat, sollte man ihn sehr umfassend studieren. In diesem Kontext sagte er: „Einen ganzen Knochen in seiner Gesamtheit zu kennen, würde beide Enden einer Unendlichkeit zusammenführen." [7]

Wiederholt ermutigte Still uns, „weiterzugraben“, um die Körperfunktionen und ihre Wechselwirkungen umfassender zu erforschen, neues Wissen einzubringen, die Os- 
teopathie am Leben zu erhalten, neue Entwicklungen und Entdeckungen in sie zu integrieren.

\section{Fazit}

In den letzten Jahrzehnten scheint der Knochen für die Wissenschaftler das aufschlussreichste Gewebe im Körper zu sein. Zudem lassen sich für uns Osteopathen alle 3 grundlegenden Axiome auf dieses Gewebe beziehen. Der Knochen spielt eine wichtige Rolle bei der Immunität. Er ist primär an der Bildung und Aufrechterhaltung der primären und sekundären Läsionen beteiligt und er sorgt dafür, dass das Blut durch die Arterien fließt, und ist gleichzeitig in der Lage, diesen Blutfluss durch Veränderungen der Körperspannung und der Kraftübertragung zu beeinflussen. Außerdem speichert der Knochen eine große Anzahl von Mineralien, die an den meisten physiologischen Prozessen beteiligt sind. Durch spezifische Zellstrukturen und ein komplexes Kanalsystem verfügt es über ein völlig eigenständiges Reaktionssystem. Und das Beste ist: Wir sind in der Lage, darauf Einfluss zu nehmen!

Wird es nicht langsam Zeit, dass wir, die Osteopathen, die Alleinstellungsmerkmale der Osteopathie in unserer alltäglichen Praxis zur Geltung kommen lassen, um so die logischste Weiterentwicklung der Medizin jener Zeit insbesondere heute in Europa in ihrer Eigenständigkeit zu bestätigen?

\section{Autorinnen/Autoren}

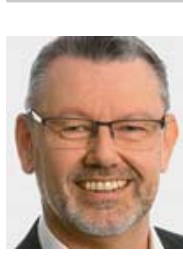

\section{Jo Buekens}

arbeitet seit 1994 als Osteopath in Luxemburg. 1999 begann er am CS, 2003 an der WSO und 2017 am JTOC zu unterrichten. 2004 erschien sein Buch Osteopathische Diagnose und Behandlung. Schwerpunkt seiner Tätigkeit und seines wissenschaftlichen Interesses sind Studien zur osteopathischen Philosophie, die angewandte Embryologie und die Knochenphysiologie. Die Ergebnisse daraus bietet er aktuell in praxisorientierten Workshops an.
Korrespondenzadresse

Jo Buekens D.O. MSc

6 , un der Mamer

8355 Garnich

Luxemburg

ostejo@pt.lu

Literatur

[1] Schmucker RV. Early Osteopathy in the words of A.T. Still. Philadelphia: Thomas Jefferson University; 1904 (Übersetzung für diesen Artikel: Jo Buekens)

[2] Booth ER. History of Osteopathy and Twentieth-Century Medical Practice. OsteoLib, Vol XIII. Kirksville: A.T. Still University; 1924 (Übersetzung für diesen Artikel: Heiko Biermann)

[3] Still AT. Autobiography of Andrew T. Still. Osteolib, Vol I. Kirksville: A.T. Still University; 1897 (Übersetzung für diese Artikel: Heiko Biermann)

[4] DiGiovanna E. An Encyclopedia of Osteopathy. Indianapolis: American Academy of Osteopathy; 2001

[5] Lane MA. A.T. Still Founder of Osteopathy. Osteolib; 1918; Vol. XIV: $15-24$

[6] Still AT. Autobiography of A.T. Still. Indianapolis: American Academy of Osteopathy, 1908 (Übersetzung für diesen Artikel Heiko Biermann)

[7] Still AT. Autobiography of Andrew T. Still with a history of the discovery and development of the science of osteopathy. Kirksville, MO; 1897 (Übersetzung für diesen Artikel Heiko Biermann)

\section{Bibliografie}

DOI https://doi.org/10.1055/a-0732-3939

DO - Deutsche Zeitschrift für Osteopathie 2019; 17: 43-46 (c) Georg Thieme Verlag KG Stuttgart · New York ISSN 1610-5044 